\title{
Investigating the Impacts of Transaction Cost under a Tradable Credit Scheme with Heterogenous Users
}

\author{
Fang Zhang $\mathbb{D}^{1,2,3}$ Jian Lu $\mathbb{C}^{1,2,3}$ Xiaojian $\mathrm{Hu}^{1,2,3}$ and Tenghui Liu ${ }^{1,2,3}$ \\ ${ }^{1}$ Jiangsu Key Laboratory of Urban ITS, Southeast University, Nanjing, China \\ ${ }^{2}$ Jiangsu Province Collaborative Innovation Center of Modern Urban Traffic Technologies, Southeast University, Nanjing, China \\ ${ }^{3}$ School of Transportation, Southeast University, Nanjing, China
}

Correspondence should be addressed to Jian Lu; lujian_1972@seu.edu.cn

Received 25 October 2020; Revised 7 December 2020; Accepted 28 January 2021; Published 16 February 2021

Academic Editor: Dazhong Ma

Copyright (C) 2021 Fang Zhang et al. This is an open access article distributed under the Creative Commons Attribution License, which permits unrestricted use, distribution, and reproduction in any medium, provided the original work is properly cited.

In this paper, the impacts of transaction cost are investigated under a tradable credit scheme (TCS) considering user heterogeneity. Under the credit scheme, a certain number of credits are initially distributed among all the travelers for a specific O-D pair, and a link-specific number of credits are charged from travelers using that link. The scheme allows for free trading of the credits among travelers, and both the sellers and buyers need to pay an extra transaction cost, which is associated with trading volume. Travelers in the network are assumed to be heterogenous with a discrete value of time (VOT). For a given tradable credit scheme and discrete VOT set, the combined network user equilibrium (UE) and credit-trading market equilibrium (ME) are formulated as a variational inequality (VI) problem, and the conditions for the uniqueness of the network flow pattern and the credit price at equilibrium are established. A bisection-based trial-and-error method is proposed to solve the proposed VI problems. Based on the simulation results, the computational advantages of the proposed method are demonstrated. Then, an example network is presented to investigate the effect of transaction cost in different kinds of markets. It is found that the implementation of transaction cost can suppress trading volume and either elevate or drive down the equilibrium credit price. Besides, it is also found that users with the lowest VOT suffer the most from the increase in transaction cost, while those with the higher VOT are more likely to experience a reduction in travel cost with the implementation of TCS.

\section{Introduction}

Tradable credit scheme (TCS) is a novel quantity-based instrument for managing traffic demand, which is designed to restrict the use of private cars. In the scheme, a central authority allocates credits to eligible travelers, and the latter need to redeem them when driving on the roads with credit charge. There is a market running under the scheme, through which credits can be freely traded. In this way, credits will flow to those with the highest value of car use (usually people with higher income), whereas those with the lower value of time (VOT) will be "pushed" out of the charging roads and get a compensation by selling their extra credits.

As an appealing alternative to the well-known road pricing scheme, TCS outperforms it in the following two aspects [1]. First, a TCS is revenue-neutral since the credit charge fees only circulate among travelers and there does not exist a direct financial transfer from travelers to the government. This can greatly enhance the public acceptability of the scheme. Second, since low-VOT users can get monetary benefits by selling their unused credits, the equity issue arising from the marginal-cost pricing scheme can be partially addressed.

1.1. Literature Review. Yang and Wang [1] first established the mathematical model of tradable credit scheme in a general network equilibrium context. From then on, a growing number of works has been devoted to the application of TCS in tackling transportation problems. Among the extensions, user heterogeneity is considered as an important issue throughout the development of TCS and incorporated by a large number of researchers. Wang et al. [2] 
extended the seminal work to a case with multiclass users and developed a TCS that can decentralize system optimal flow patterns in such a heterogenous context. Later, Zhu et al. [3] generalized the groups with discrete VOTs into a continuously distributed VOT and also developed a system optimal TCS. With a continuously distributed VOT, Tian et al. [4] examined the efficiency of TCS for managing bottleneck congestion and modal split in a competitive highway/transit network with a continuously distributed VOT. More recently, Wang et al. [5] proposed a tradable O-D-based travel credit scheme to manage mobility in a bimodal transportation network with heterogenous users in VOT. Miralinaghi and Peeta [6] focused on TCS-based multiperiod equilibrium modeling framework to minimize the vehicular emissions in a traffic network over a planning horizon, considering multiclass users with different VOTs. Also, Wu et al. [7] and $\mathrm{He}$ et al. [8] considered the user heterogeneity in terms of different income levels and path choice behaviors in the context of TCS, respectively. Miralinaghi et al. [9] considered commuters' heterogeneities in terms of value-of-time, schedule delay, and loss aversion behavior in purchasing credits and showed the impact of initial credit allocation in a dynamic context.

Besides, Shirmohammadi et al. [10] established the identity between congestion pricing and tradable credit schemes in managing network and demonstrated how the identity fell apart when uncertainty in transportation supply or demand was taken into account. Later, the authors proposed a credit scheme to control the maximum queue length at a bottleneck [11]. Wang et al. [12] proposed a bilevel programming model for continuous network design problem with TCS and equity constraints. Miralinaghi and Peeta [13] proposed a multiperiod TCS, in which travelers determined whether to consume or sell their credits in the current period or to transfer to future periods. Based on the multiperiod TCS, the authors moved on to minimize the vehicular emissions in a traffic network over a planning horizon [6, 14]. Wang and Zhang [15] investigated joint implementation of tradable credit and road pricing in public-private partnership (PPP) networks by simultaneously taking into account Cournot-Nash (CN) players and user equilibrium (UE) players. Also, in PPP networks, Bao et al. [16] employed the TCS on traffic mobility management through a novel kind of private financing of public road, build-equity-credit (BEC) scheme, hoping to achieve a triple win for government, private firms, and travelers. Combining TCS and link capacity improvement, Wang et al. [17] proposed a biobjective bilevel programming model to balance economic growth and environmental management. Integrating link-based discrete credit charging scheme into the discrete network design problem, Wang et al. [18] proposed a mixed-integer nonlinear bilevel programming model to improve the network performance from the perspectives of both transport network planning and travel demand management. For autonomous vehicle (AV) management, Zhang et al. [19] investigated the traffic equilibriums for mixed traffic flows of human-driven vehicles and connected autonomous vehicles in the context of TCS, and Seilabi et al. [20] developed a scheme for travel demand and lane management strategies in the AV transition era. There are also some works including a case study based on experiments [21-24]. More detailed recent reviews in be found in $[25,26]$. All of these works fulfill the framework of TCS and provide good inspirations for this paper.

1.2. Research Objectives and Contributions. In the literature, most of the works presumed that transaction costs were "low enough" and ignorable in their framework, citing the fact that transaction may be made using affordable electronic technologies at low costs. However, as demonstrated in Nie [27], the transaction cost incurred during credit trading may not be reduced to a negligible level even with an ideal, fully computerized system. It would still take time to find and match potential buyers and sellers within the credit-trading market. The ignorance of transaction cost can lead to an underestimate on actual travel cost and thus a totally different flow pattern.

To the best of the authors' knowledge, only four papers considering transaction cost in a TCS can be found. Nie [27] established an optimization model that incorporates transaction cost and investigated the performance of various combinations of the credit price, the government price, and the marginal transaction cost. He et al. [8] examined the effects of transaction cost on a network with Cournot-Nash players and Wardrop-equilibrium players, in which different forms of transaction cost are adopted for different types of users. Bao et al. [28] focused on the equilibrium status and the optimal design of TCS when transaction cost as well as travelers' loss aversion is considered. More recently, Zhang et al. [29] investigated the impact of transaction cost on social equity in terms of different user classes. All the four papers adopted a linear function of transaction cost. However, it may not be the case in reality. As Cason and Gangadharan put it [30], a decreasing marginal transaction cost can occur if any discount is offered over quantity or people become familiar with the market and can match their trading partners with less searching and information costs. Similarly, an increasing marginal cost can occur when the scheme is initially implemented and people need to make more effort to search and find a better trading partner to trade more credits [31]. Given the fact that different forms of transaction cost may affect the system in different ways, in this paper, we investigate the impacts of three typical forms of transaction costs under a TCS considering user heterogeneity.

The contributions of this paper are threefold. First, we incorporate user heterogeneity as well as transaction cost into a variational inequality (VI) model and establish the conditions for the uniqueness of the network flow pattern and the credit price at equilibrium. Second, a novel bisection-based trial-and-error method is proposed to solve the proposed VI problem and its convergence to the equilibrium solution is mathematically proved. Third, we assume three different types of transaction costs and numerically investigate the impacts of these transaction costs on the credit market and individual better-off degree under various 
settings, which can provide managerial insights for markets with different operate mechanisms.

The remainder of this paper is organized as follows. In Section 2, we analyze the UE and ME under a credit scheme considering transaction cost. Then, an equivalent variational inequality (VI) formulation is presented and the uniqueness of solutions is demonstrated. In Section 3, the theoretical conditions for decentralizing SO flow pattern are discussed. In Section 4, we propose a solution algorithm for the problem and present the details on it. In Section 5, we present example networks to test the computational efficiency of the proposed algorithm and examine the impacts of transaction cost under a given credit scheme. Eventually, we present concluding remarks and recommendations for future research in Section 6.

\section{Traffic Equilibrium under a Given Tradable Credit Scheme considering Transaction Cost}

2.1. Tradable Credit Scheme with Transaction Cost. Let $G=$ $(N, A)$ be a general transportation network defined by a set of nodes $N$ and a set of directed links $A$. Let $W$ denote the set of O-D pairs and $R^{w}$ denote the set of all paths connecting O-D pair $w \in W$. A discrete set of user classes in terms of VOT, denoted by $M$, is assumed in this paper. Let $\beta^{m}$ denote the average VOT for users of class $m \in M$, and further $\beta=\left(\beta^{m}, m \in M\right)$. The travel demand is assumed to be given and fixed, denoted by a vector $\left(d_{w}^{m}>0, w \in W, m \in M\right)^{T}$, and $d_{w}^{m}$ is the demand of user class $m \in M$ within O-D pair $w \in W$. The flow of user class $m \in M$ on path $r \in R^{w}$ is denoted by $f_{r, w}^{m}$. A separable link travel time function, $t_{a}\left(v_{a}\right)$, which is strictly convex and monotonically increasing with the aggregate link flow $v_{a}$ on link $a \in A$, is assumed. The class-specific link flow $v_{a}^{m}$ and the aggregate link flow $v_{a}$ on link $a \in A$ have the following relationships with the path flow:

$$
\begin{aligned}
v_{a}^{m} & =\sum_{w \in W} \sum_{r \in R^{w}} f_{r, w}^{m} \delta_{a, r}^{w}, \quad a \in A, m \in M, \\
v_{a} & =\sum_{m \in M} v_{a}^{m}, \quad a \in A
\end{aligned}
$$

where $\delta_{a, r}^{w}=1$ if link $a$ belongs to path $r$ and 0 otherwise. For notational simplicity, we denote path and link flows in vectors as $f^{M}=\left(f_{r, w}^{m}, r \in R^{w}, w \in W, m \in M\right)^{T}$, $v=\left(v_{a}, a \in A\right)^{T}$, and $v_{a}^{M}=\left(v_{a}^{m}, a \in A, m \in M\right)^{T}$. The feasible set of flow patterns $\left(f^{M}, v^{M}, v\right)$ can be defined by

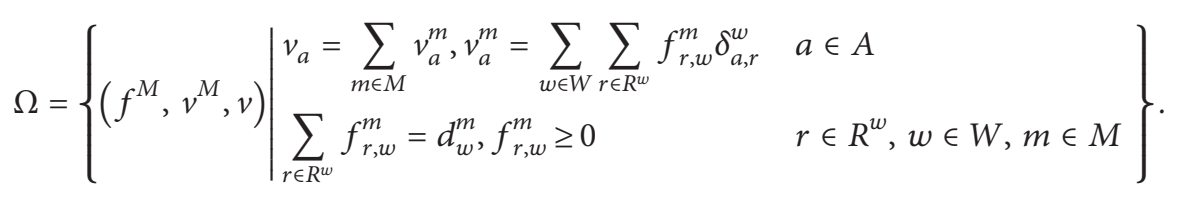

In this paper, we adopt an O-D-specific allocation of credits at the initial stage. Denote $\varphi=\left(\varphi_{w}, w \in W\right)^{T}$ as the general $\mathrm{O}-\mathrm{D}$-specific but user-anonymous credit allocation scheme, where $\phi_{w}$ is the amount of credits distributed to travelers within O-D pair $w \in W$. Let $K$ denote the total amount of distributed credits, satisfying with $K=\sum_{w \in W} \sum_{m \in M} \varphi_{w} d_{w}^{m}$. The link-specific credit charging scheme is denoted by $\kappa=\left(\kappa_{a}, a \in A\right)^{T}$, where $\kappa_{a}$ is the credit charge for any traveler who uses link $a \in A$. We use $(K, \kappa)$ to characterize a credit charge scheme $\kappa$ under a total number of credits $K$ issued in the market.

Further, we define the feasible set of credit schemes that guarantees the existence of equilibrium flow patterns as $\Psi$, which is given by

$$
\psi=\left\{(K, \kappa) \mid \exists\left(f^{M}, v^{M}, v\right) \in \Omega \text { such that } \sum_{a \in A} \kappa_{a} v_{a} \leq K\right\} .
$$

Moreover, we assume that both sellers and buyers need to pay the same amount of additional cost in a transaction (through trading credits, sellers gain monetary benefit by giving up their trips or choosing alternative mode of transport, and buyers pay for excess demand of credits but benefit from the congestion relief; therefore, either more monetary benefit or less travel time, every traveler gets what they want and neither buyers nor sellers need to be further compensated; from the standpoint of equity, we assume that both sellers and buyers need to pay the same transaction cost in this paper). The transaction cost, $T_{r}^{w}$, is defined as a function of trading volume $\left|e_{r}^{w}\right|$.

$$
T_{r}^{w}=T_{r}^{w}\left(\left|e_{r}^{w}\right|\right)=\rho \cdot\left|e_{r}^{w}\right|^{\eta}, \quad r \in R^{w}, w \in W,
$$

where $\rho$ is a proportionality constant and $\eta$ represents the function form of transaction cost, which depends on the market operating mechanism. In particular, $\eta<1$ implies a decreasing marginal transaction cost, which can occur if the broker offers discount over quantity or travelers become more experienced with the market and can thus fulfill their credit demands with less searching and information costs, and we refer to this market as a "trading-encouraging" one; $\eta>1$ implies an increasing marginal cost, which can be justified by the fact that travelers may have to make more effort to search and find a better trading partner to trade more credits, and we refer to this market as a "trading-controlling" one, and $\eta=1$ implies a linear function with constant marginal cost, indicating that the difficulty in trading credits does not change with the trading volume; we refer to this market as a moderate one.

The term $e_{r}^{w}$ here is defined by

$$
e_{r}^{w}=\sum_{a \in A} k_{a} \delta_{a, r}^{w}-\varphi_{w}=k_{r}^{w}-\varphi_{w}, \quad r \in R^{w}, w \in W,
$$

where $\kappa_{r}^{w}=\sum_{a \in A} \kappa_{a} \delta_{a, r}^{w}$. Since the charged credits are equal to the issued at equilibrium, the total trading volume (TV) 
should be the extra credit demands for all the travelers on paths with $e_{r}^{w}>0$, i.e.,

$$
\mathrm{TV}=\sum_{w \in W} \sum_{r \in R_{+}^{w}} e_{r}^{w} f_{r}^{w}
$$

where $R_{+}^{w}=\left\{r \in R^{w} \mid e_{r}^{w}>0\right\}$.

Based on the assumptions above, the generalized travel cost (travel disutility) for each traveler consists of three parts: (1) travel time in monetary unit; (2) credit cost; and (3) transaction cost. The generalized travel cost for user class $m$ using path $r$ between O-D pair $w$ is given by

$$
c_{r, w}^{m}=\sum_{a \in A} \beta^{m} t_{a}\left(v_{a}\right) \delta_{a, r}^{w}+p e_{r}^{w}+T_{r}^{w}, \quad r \in R^{w}, w \in W, m \in M,
$$

where $p$ denotes the unit credit price. The credit cost for using a path $r \in R^{w}$ is given by the gap between the charge and initial distribution credits, $e_{r}^{w}$, multiplied by the unit credit price, $p$. Similarly, this term can be either positive or negative, representing a cost or a reward, respectively.

2.2. Variational Inequalities Formulation. Since the credit $\operatorname{cost} e_{r}^{w}$ and the transaction cost $T_{r}^{w}$ are nonadditive of link costs, it is technically hard to describe the problem by a link-based optimization model. In the literature, Nie [27] and $\mathrm{He}$ et al. [8] addressed this difficulty by introducing a new variable, $g_{r}^{w}$, which represents the travelers who choose to trade their credits in the market on path $r$ connecting $\mathrm{O}-\mathrm{D}$ pair $w$, into the minimization program and incorporating the total transaction cost into the objective function. However, Zhang et al. [29] proved that the equilibrium solution of this variable is nonunique, meaning that the credit trading in the market is unpredictable. By comparison, a path-based optimization model can incorporate the nonadditive cost without adding any new variable. Actually, Bao et al. [28] successfully formulated the combined network user equilibrium (UE) and credittrading market equilibrium (ME) considering transaction cost as a variational inequality (VI) formulation. Therefore, a VI formulation is also adopted in this paper. Different from [28], we further consider the user heterogeneity in terms of VOT in the model.

Let $p^{*}$ denote the unit credit price at credit market equilibrium and $\left(f^{M}, v^{M}, v\right)$ denote the UE flow pattern. Then, given a feasible credit scheme $(K, \kappa) \in \Psi$, the UE conditions can be written as

$$
\begin{aligned}
& \sum_{a \in A} \beta^{m} t_{a}\left(v_{a}^{*}\right) \delta_{a, r}^{w}+p^{*} e_{r}^{w}+T_{r}^{w}=\mu_{w}^{m *}, \quad \text { if } f_{r, w}^{m *}>0, r \in R^{w}, w \in W, m \in M, \\
& \sum_{a \in A} \beta^{m} t_{a}\left(v_{a}^{*}\right) \delta_{a, r}^{w}+p^{*} e_{r}^{w}+T_{r}^{w}=\mu_{w}^{m *}, \quad \text { if } f_{r, w}^{m *}=0, r \in R^{w}, w \in W, m \in M,
\end{aligned}
$$

and the credit ME conditions are given by

$$
\begin{aligned}
& \sum_{a \in A} \kappa_{a} v_{a}^{*}=K, \quad \text { if } p^{*}>0, \\
& \sum_{a \in A} \kappa_{a} v_{a}^{*} \leq K, \quad \text { if } p^{*}=0,
\end{aligned}
$$

where $\left(f^{M *}, v^{M *}, v\right) \in \Omega$. Equations (7) and (8) imply that, at UE equilibrium, all utilized paths by user class $m \in M$ between O-D pair $w \in W$ have equal and minimal generalized travel cost $\mu_{w}^{m *}$ in monetary unit. Meanwhile, equations (10) and (11) represent the credit market clearing conditions, which imply that the equilibrium credit price is positive only if all the issued credits are consumed.

In the following proposition, we show how to obtain the $\mathrm{UE}$ and ME solutions under a given credit scheme.

Proposition 1. Given a tradable credit scheme $(K, \kappa) \in \psi,\left(f^{M *}, v^{M *}, v\right) \in \widetilde{\Omega}(K, \kappa)$ is the aggregate $U E$ flow pattern if and only if it solves the following VI problem:

$$
\begin{aligned}
& \sum_{w \in W} \sum_{r \in R^{w}} \sum_{m \in M}\left(\sum_{a \in A} \beta^{m} t_{a}\left(v_{a}^{*}\right) \delta_{a, r}^{w}+p * e_{r}^{w}+T_{r}^{w}\right)\left(f_{r, w}^{m}-f_{r, w}^{*}\right) \\
& +\left(\sum_{a \in A} \kappa_{a} v_{a}^{*}-K\right)\left(p-p^{*}\right) \geq 0, \quad \forall\left(f^{M}, v^{M}, v\right) \in \widetilde{\Omega}(K, \kappa), \forall p \geq 0,
\end{aligned}
$$

where

$$
\widetilde{\Omega}(K, \kappa)=\left\{\left(f^{M}, v^{M}, v\right) \mid \sum_{a \in A} \kappa_{a} v_{a} \leq K, \quad\left(f^{M}, v^{M}, v\right) \in \Omega\right\} \in \Omega,
$$


and the equilibrium credit price, $p^{*}$, corresponds to the Lagrange multiplier associated with the credit conservation constraint:

$$
\sum_{a \in A} \kappa_{a} v_{a} \leq K
$$

$$
\begin{aligned}
& \sum_{w \in W} \sum_{r \in R^{w}} \sum_{m \in M}\left(\sum_{a \in A} \beta^{m} t_{a}\left(v_{a}^{*}\right) \delta_{a, r}^{r}+p^{*} e_{r}^{w}+T_{r}^{w}\right) f_{r, w}^{m *}+\left(\sum_{a \in A} \kappa_{a} v_{a}^{*}\right) p^{*} \\
& \leq \sum_{w \in W} \sum_{r \in R^{w}} \sum_{m \in M}\left(\sum_{a \in A} \beta^{m} t_{a}\left(v_{a}^{*}\right) \delta_{a, r}^{w}+p^{*} e_{r}^{w}+T_{r}^{w}\right) f_{r, w}^{m} \\
& +\left(\sum_{a \in A} \kappa_{a} v_{a}^{*}-K\right), \quad \forall\left(f^{M}, v^{M}, v\right) \in \widetilde{\Omega}(K, \kappa), \forall p \geq 0 .
\end{aligned}
$$

So, $\left(f^{M}, v^{M}, v\right) \in \widetilde{\Omega}(K, \kappa)$ solves VI problem (12) if and only if it solves the following optimization problem [32]:

$$
\min _{\left(f^{M}, v^{M}, v\right), p} \sum_{w \in W} \sum_{r \in R} \sum_{m \in M}\left(\sum_{a \in A} \beta^{m} t_{a}\left(v_{a}^{*}\right) \delta_{a, r}^{w}+p * e_{r}^{w}+T_{r}^{w}\right) f_{r, w}^{m}+\left(\sum_{a \in A} \kappa_{a} v_{a}^{*}-K\right) p
$$

subject to

$$
\left(f^{M}, v^{M}, v\right) \in \widetilde{\Omega}(K, \kappa), \quad p \geq 0,
$$

with $v^{*}$ fixed and $\widetilde{\Omega}(K, \kappa)$ being a polyhedron; the above optimization problem is a linear program. The Lagrangian function is

$$
\begin{aligned}
L= & \sum_{w \in W} \sum_{r \in R} \sum_{m \in M}\left(\sum_{a \in A} \beta^{m} t_{a}\left(v_{a}^{*}\right) \delta_{a, r}^{w}+p^{*} e_{r}^{w}+T_{r}^{w}\right) f_{r, w}^{m} \\
& +\left(\sum_{a \in A} \kappa_{a} v_{a}^{*}-K\right) p-\sum_{w \in W} \sum_{m \in M} \mu_{w}^{m}\left(\sum_{r \in R^{w}} f_{r, w}^{m}-d_{w}^{m}\right) .
\end{aligned}
$$

The Karush-Kuhn-Tucker (KKT) conditions for this problem are

$$
\begin{gathered}
f_{r, w}^{m *}\left(\sum_{a \in A} \beta^{m} t_{a}\left(v_{a}^{*}\right) \delta_{a, r}^{w}+p^{*} e_{r}^{w}+T_{r}^{w}-\mu_{w}^{m}\right)=0, \quad r \in R^{w}, w \in W, m \in M, \\
\sum_{a \in A} \beta^{m} t_{a}\left(v_{a}^{*}\right) \delta_{a, r}^{w}+p^{*} e_{r}^{w}+T_{r}^{w}-\mu_{w}^{m} \geq 0, \quad r \in R^{w}, w \in W, m \in M, \\
f_{r, w}^{m *} \geq 0, \quad r \in R^{w}, w \in W, m \in M, \\
\sum_{r \in R^{w}} \sum_{m \in M} f_{r, w}^{m *}=d_{w}^{m}, \quad w \in W,
\end{gathered}
$$




$$
\begin{aligned}
&\left(\sum_{a \in A} \kappa_{a} v_{a}^{*}-K\right) p^{*}=0, \\
& \sum_{a \in A} \kappa_{a} v_{a}^{*}-K \leq 0, \quad p^{*} \geq 0 .
\end{aligned}
$$

Obviously, the above conditions (19), (20) and (23), (24) are equivalent to the user equilibrium conditions (8), (9) and the credit market equilibrium conditions (10), (11). Condition (21) represents the nonnegative path flows and condition (22) represents the flow conservation. The Lagrange multipliers $p^{*}$ and $\mu_{w}^{m}, w \in W$, and $m \in M$ correspond to the unit credit price at market equilibrium and the minimal generalized travel cost for O-D pair $w$ for user class $m$ at user equilibrium. This completes the proof.

Proposition 2. There exists at least one solution to VI problem (12).

Proof. Due to the convexity and nonemptiness of the feasible set $\widetilde{\Omega}(K, \kappa)$ and the continuity of the generalized travel cost $\sum_{a \in A} \beta^{m} t_{a}\left(v_{a}^{*}\right) \delta_{a, r}^{w}+p^{*} e_{r}^{w}+T\left(\left|e_{r}^{w}\right|\right), r \in R^{w}$, $w \in W, m \in M$ and $\sum_{a \in A} \kappa_{a} v_{a}^{*}-K$ with respect to path flow pattern $f^{M}$ and credit price $p$, we can conclude based on [32] that at least one solution $\left(f^{M *}, v^{M *}, v^{*}\right)$ and $p^{*}$ satisfying VI problem (12) exists.

2.3. Uniqueness of the Link Flow Pattern and Equilibrium Credit Price. Then, we move on to establish the uniqueness condition for the UE flow pattern and corresponding credit price solved by VI problem (12).

Before that, the following lemma is needed, since the proposed VI formulation does not coincide with any optimization problem due to user heterogeneity.
Lemma 1. Let $\left(f^{M *}, v^{M *}, v^{*}\right)$ and $p^{*}$ denote the solution of $V I$ problem (12); then, at equilibrium, $\sum_{w \in W} \sum_{r \in R^{w}}$ $\sum_{m \in M}\left(\sum_{a \in A} \beta^{m} t_{a}\left(v_{a}^{*}\right) \delta_{a, r}^{w}+p^{*} e_{r}^{w}+T_{r}^{w}\right)\left(f_{r, w}^{m}-f_{r, w}^{m *}\right) \geq 0$.

Proof. If $p^{*}=0$, from inequality (15), we have

$$
\begin{aligned}
& \sum_{w \in W} \sum_{r \in R^{w}} \sum_{m \in M}\left(\sum_{a \in A} \beta^{m} t_{a}\left(v_{a}^{*}\right) \delta_{a, r}^{w}+p^{*} e_{r}^{w}+T_{r}^{w}\right)\left(f_{r, w}^{m}-f_{r, w}^{m *}\right) \\
& \geq\left(K-\sum_{a \in A} \kappa_{a} v_{a}^{*}\right) p, \quad \forall\left(f^{M}, v^{M}, v\right) \in \widetilde{\Omega}(K, \kappa), \forall p \geq 0 .
\end{aligned}
$$

Since $\left(f^{M *}, v^{M *}, v^{*}\right) \in \widetilde{\Omega}(\mathrm{K}, \kappa)$, we have $\sum_{a \in A} \kappa_{a} v_{a}^{*} \leq K$, adding that $p \geq 0$; it can be referred that $\left(K-\sum_{a \in A} \kappa_{a} v_{a}^{*}\right) p \geq 0$. Thus, the left-hand-side term in the above inequality must be equal or greater than zero, i.e.,

$$
\sum_{w \in W} \sum_{r \in R^{w}} \sum_{m \in M}\left(\sum_{a \in A} \beta^{m} t_{a}\left(v_{a}^{*}\right) \delta_{a, r}^{w}+p^{*} e_{r}^{w}+T_{r}^{w}\right)\left(f_{r, w}^{m}-f_{r, w}^{m *}\right) \geq 0 .
$$

If $p^{*} \neq 0$, then at equilibrium, the market equilibrium condition must be satisfied, i.e., $\sum_{a \in A} \kappa_{a} v_{a}^{*}-K$. Since $\left(f^{M *}, v^{M *}, v^{*}\right)$ is the solution of VI problem (12), we have

$$
\begin{aligned}
& \sum_{w \in W} \sum_{r \in R^{w}} \sum_{m \in M}\left(\sum_{a \in A} \beta^{m} t_{a}\left(v_{a}^{*}\right) \delta_{a, r}^{w}+p^{*} e_{r}^{w}+T_{r}^{w}\right)\left(f_{r, w}^{m}-f_{r, w}^{m *}\right)+\left(\sum_{a \in A} \kappa_{a} v_{a}^{*}-K\right) \\
& \quad\left(p-p^{*}\right)=\sum_{w \in W} \sum_{r \in R^{w}} \sum_{m \in M}\left(\sum_{a \in A} \beta^{m} t_{a}\left(v_{a}^{*}\right) \delta_{a, r}^{w}+p^{*} e_{r}^{w}+T_{r}^{w}\right)\left(f_{r, w}^{m}-f_{r, w}^{m *}\right) \geq 0, \quad \forall\left(f^{M}, v^{M}, v\right) \in(K, \kappa), \forall p \geq 0 .
\end{aligned}
$$

Thus, we can say that the inequality $\sum_{w \in W} \sum_{r \in R^{w}} \sum_{m \in M}\left(\sum_{a \in A} \beta^{m} t_{a}\left(v_{a}^{*}\right) \delta_{a, r}^{w}+p^{*} e_{r}^{w}+T_{r}^{w}\right)\left(f_{r, w}^{m}-\right.$ $\left.f_{r, w}^{m *}\right) \geq 0$ holds at equilibrium. This completes the proof.

With Lemma 1, we now can give the sufficient condition for the uniqueness of the link flow pattern. The proof process follows that of [28], originating from [33].

Proposition 3. Given a tradable credit scheme $(K, \kappa) \in \Psi$, if $t$ $(v)$ is strictly weighted average monotone over $\beta$ on $\widetilde{\Omega}(K, \kappa)$ [2], that is,

$$
\sum_{a \in A} \sum_{m \in M} \beta^{m}\left(t_{a}\left(v_{a}^{\prime}\right)-t_{a}\left(v_{a}^{\prime \prime}\right)\right)\left(v_{a}^{m^{\prime}}-v_{a}^{m^{\prime \prime}}\right)>0
$$

then the aggregate UE link flow pattern $v^{*}$ is unique.

Proof. Suppose two solution flow patterns and corresponding credit prices $\left(f^{M^{\prime}}, v^{M^{\prime}}, v^{\prime}\right) \in \widetilde{\Omega}(K, \kappa), p^{\prime} \geq 0$ and $\left(f^{M^{\prime \prime}}, v^{M^{\prime \prime}}, v^{\prime \prime}\right) \in \widetilde{\Omega}(K, \kappa), p^{\prime \prime} \geq 0$ with $v^{\prime} \neq v^{\prime \prime}$ to VI problem (12); then, by setting $\left(f^{M *}, v^{M *}, v^{*}\right) \in\left(f^{M^{\prime}}, v^{M^{\prime}}, v^{\prime}\right), p^{*}=$ $p^{\prime}$ and $\left(f^{M}, v^{M}, v\right)=\left(f^{M^{\prime \prime}}, v^{M^{\prime \prime}}, v^{\prime \prime}\right), p=p^{\prime \prime}$, we have 


$$
\sum_{w \in W} \sum_{r \in R^{w}} \sum_{m \in M}\left(\sum_{a \in A} \beta^{m} t_{a}\left(v_{a}^{\prime}\right) \delta_{a, r}^{w}+p^{\prime} e_{r}^{w}+T_{r}^{w}\right)\left(f_{r, w}^{\prime \prime}-f_{r, w}^{\dot{m}}\right) \geq 0 .
$$

By exchanging $\left(f^{M^{\prime}}, v^{M^{\prime}}, v^{\prime}, p^{\prime}\right)$ and $\left(f^{M^{\prime \prime}}, v^{M^{\prime \prime}}, v^{\prime \prime}\right.$, $p^{\prime \prime}$ ), we have

$$
\sum_{w \in W} \sum_{r \in R^{w}} \sum_{m \in M}\left(\sum_{a \in A} \beta^{m} t_{a}\left(v_{a}^{\prime \prime}\right) \delta_{a, r}^{w}+p^{\prime \prime} e_{r}^{w}+T_{r}^{w}\right)\left(f_{r, w}^{\dot{m}}-f_{r, w}^{\prime \prime m}\right) \geq 0 .
$$

Adding equations (29) and (30) yields

$$
\begin{aligned}
& \sum_{a \in A} \sum_{m \in M} \beta^{m}\left(t_{a}\left(v_{a}^{\prime}\right)-t_{a}\left(v_{a}^{\prime \prime}\right)\right)\left(v_{a}^{\dot{m}^{\prime}}-v_{a}^{m^{\prime \prime}}\right) \\
& \quad+\sum_{w \in W} \sum_{r \in R^{w}} \sum_{m \in M}\left(p^{\prime}-p^{\prime \prime}\right) e_{r}^{w}\left(f_{r, w}^{\dot{m}}-f_{r, w}^{\prime \prime}\right) \geq 0 .
\end{aligned}
$$

It should be noted that at equilibrium, the total credits sold must be equal to the total credits bought at the credit market, that is,

$$
\sum_{w \in W} \sum_{r \in R^{w}} \sum_{m \in M} e_{r}^{w} f_{r, w}^{m}=0, \quad \forall\left(f^{m}, v^{M}, v\right) \in \widetilde{\Omega}(K, \kappa) .
$$

Then, inequality (31) can be transformed into

$$
\sum_{a \in A} \sum_{m \in M} \beta^{m}\left(t_{a}\left(v_{a}^{\prime \prime}\right)-t_{a}\left(v_{a}^{\prime}\right)\right)\left(v_{a}^{\prime}-v_{a}^{\prime \prime}\right) \geq 0
$$

which clearly contradicts inequality (27). Therefore, the aggregate UE link flow pattern must be unique if $t(v)$ is strictly weighted average monotone over $\beta$ on $\widetilde{\Omega}(K, \kappa)$. This completes the proof.

With the uniqueness of flow pattern, we can further establish the conditions for unique equilibrium credit price.

Proposition 4. Given a tradable credit scheme $(K, \kappa) \in \Psi$, the equilibrium credit price $p^{*}$ is unique if (1) $v^{*}$ is unique and (2) among all the corresponding UE path flow patterns $f^{M *}$, there exists at least one user class $m$ whose equilibrium path set always contains the same two (or more) paths connecting one O-D pair with different credit charges.

Proof. It should be noted that with a given link flow pattern $v^{*}$, the uniqueness of path flow pattern $f^{M *}$ cannot be ensured. In other words, even if $v^{*}$ is unique, $f^{M *}$ is generally not unique. We define $\Omega_{f}^{M *}$ as the set of all UE path flow patterns $f^{M *}$. Accordingly, it is assumed that for any $f^{M *} \in \Omega_{f}^{M *}$, the equilibrium path set of a user class $m$ always contains two paths, $r_{1}, r_{2} \in R^{w}$ connecting O-D pair $w$ and $\kappa_{r_{1}}^{w} \neq \kappa_{r_{2}}^{w}$. Since $e_{r}^{w}=\kappa_{r}^{w}-\varphi_{w}$, it is clear that $e_{r_{1}}^{w} \neq e_{r_{2}}^{w}$. From UE condition (8), we have

$$
\begin{aligned}
& \sum_{a \in A} \beta^{m} t_{a}\left(v_{a}^{*}\right) \delta_{a, r_{1}}^{w}+p^{*} e_{r_{1}}^{w}+T_{r_{1}}^{w}=\mu_{w}^{m *}, \\
& \sum_{a \in A} \beta^{m} t_{a}\left(v_{a}^{*}\right) \delta_{a, r_{2}}^{w}+p^{*} e_{r_{2}}^{w}+T_{r_{2}}^{w}=\mu_{w}^{m *} .
\end{aligned}
$$

Subtracting equation (35) by equation (34) yields

$$
p^{*}=\frac{\sum_{a \in A} \beta^{m} t_{a}\left(v_{a}^{*}\right)\left(\delta_{a, r_{1}}^{w}-\delta_{a, r_{2}}^{w}\right)+T_{r_{1}}^{w}-T_{r_{2}}^{w}}{e_{r_{2}}^{w}-e_{r_{1}}^{w}} .
$$

Apparently, if $v^{*}$ is unique, $p^{*}$ can be uniquely determined by equation (36). This completes the proof.

Accordingly, the uniqueness of credit price cannot be ensured when there is only one path connecting each O-D pair, as exemplified by Yang and Wang [1]. However, the uniqueness conditions are easy to achieve in small network and much easier in large-size network in reality due to the multiple paths connecting each O-D pair.

In the above discussion, it is demonstrated that the proposed VI problem has a unique solution related to link flow pattern and credit price. However, due to the existence of market equilibrium price involved in the travel disutility credit the credit market clearing conditions as constraints, it is a challenge for traditional solution methods like Frank-Wolfe to solve the problem. Thus, we propose a new solution algorithm for this problem, which is introduced in the next section.

\section{Tradable Credit Scheme Design considering Transaction Cost}

As shown in [2], in the absence of transaction cost, a given SO link flow pattern $\widetilde{v}$, either cost-based or time-based, can be decentralized by any tradable credit scheme $(\widetilde{K}, \widetilde{\kappa})$ contained in the following nonempty polyhedron with $\mathrm{ME}$ credit price $p^{*}=1$ :

$$
\begin{gathered}
\sum_{a \in A} \sum_{m \in M} \beta^{m} t_{a}\left(\widetilde{v}_{a}\right) \delta_{a, r}^{w}+\sum_{a \in A} \widetilde{\kappa}_{a} \widetilde{v}_{a}=\sum_{w \in W} \sum_{m \in M} \mu_{w}^{m} d_{w}^{m}, \\
\sum_{m \in M} \beta^{m} t_{a}\left(\widetilde{v}_{a}\right) \delta_{a, r}^{w}+\sum_{a \in A} \widetilde{\kappa}_{a} \delta_{a, r}^{w} \geq \mu_{w}^{m}, \quad r \in R^{w}, w \in W, m \in M .
\end{gathered}
$$

Based on the above knowledge on credit scheme design, we can give the sufficient conditions for decentralizing SO flow pattern under a tradable credit scheme considering transaction cost.

Proposition 5. Assume the credit scheme $(\widetilde{K}, \widetilde{\kappa})$ is determined by conditions (37) and (38) and $t(v)$ is strictly weighted average monotone over $\beta$ on $\widetilde{\Omega}(\widetilde{K}, \widetilde{\kappa})$. The solution of VI problem (12) can be decentralized into SO flow pattern only when the following conditions are satisfied: (1) among all the corresponding UE path flow patterns $f^{M *}$, there exists at least one user class $m$ whose equilibrium path set always contains the same two (or more) paths $r_{1}$ and $r_{2}$ connecting one O-D pair $w$ with different credit charges and (2) the charges satisfy $\widetilde{\kappa}_{r_{1}}^{w}+\widetilde{\kappa}_{r_{2}}^{w}=2 \varphi_{w}$.

Proof. The strict weighted average monotonicity and the first condition are assumed for the uniqueness of the 
aggregate UE flow pattern and ME credit price, respectively. With $p^{*}=1,(\widetilde{K}, \widetilde{\kappa})$ can be easily proved to be the target scheme. However, due to the existence of transaction cost, the condition $p^{*}=1$ cannot be ensured. To make it hold and decentralize the SO flow pattern, $\widetilde{\kappa}_{r_{1}}^{w}+\widetilde{\kappa}_{r_{2}}^{w}=2 \varphi_{w}$ should be satisfied for the two related paths. The reason is given below.

Since $(\widetilde{K}, \widetilde{\kappa})$ is determined by conditions (37) and (38), we have

$$
\begin{aligned}
& \sum_{a \in A} \beta^{m} t_{a}\left(\widetilde{v}_{a}\right) \delta_{a, r_{1}}^{w}+\widetilde{\kappa}_{r_{1}}^{w}=\sum_{a \in A} \beta^{m} t_{a}\left(\widetilde{v}_{a}\right) \delta_{a, r_{2}}^{w}+\widetilde{\kappa}_{r_{2}}^{w}, \\
& \sum_{a \in A} \beta^{m} t_{a}\left(\widetilde{v}_{a}\right)\left(\delta_{a, r_{1}}^{w}-\delta_{a, r_{2}}^{w}\right)=\widetilde{\kappa}_{r_{2}}^{w}-\widetilde{\kappa}_{r_{1}}^{w}=e_{r_{2}}^{w}-e_{r_{1}}^{w} .
\end{aligned}
$$

When SO is achieved, we have

$$
\begin{aligned}
p^{*} & =\frac{\sum_{a \in A} \beta^{m} t_{a}\left(\widetilde{v}_{a}\right)\left(\delta_{a, r_{1}}^{w}-\delta_{a, r_{2}}^{w}\right)+T_{r_{1}}^{w}-T_{r_{2}}^{w}}{e_{r_{2}}^{w}-e_{r_{1}}^{w}} \\
& =1+\frac{T_{r_{1}}^{w}-T_{r_{2}}^{w}}{e_{r_{2}}^{w}-e_{r_{1}}^{w}} .
\end{aligned}
$$

From the second condition, we can get

$$
\widetilde{\kappa}_{r_{1}}^{w}-\varphi_{w}=-\left(\kappa_{r_{1}}^{w}-\varphi_{w}\right)
$$

namely,

$$
\left|e_{r_{1}}^{w}\right|=\left|e_{r_{2}}^{w}\right|
$$

and it follows readily that

$$
T_{r_{1}}^{w}=T_{r_{2}}^{w}
$$

Then, we have

$$
p^{*}=1+\frac{T_{r_{1}}^{w}-T_{r_{2}}^{w}}{e_{r_{2}}^{w}-e_{r_{1}}^{w}}=1 .
$$

Considering the other conditions above, we can conclude that the solution of VI problem (12) can be decentralized into SO flow pattern. This completes the proof.

With the two conditions in Proposition 5 satisfied, the tradable credit scheme achieving SO flow pattern can be determined. As mentioned before, the first condition can be always ensured in realistic network; however, the second condition is rather difficult to achieve in practice. This is because the exact two paths for calculation of credit price can be only determined endogenously based on the resultant flow pattern. In other words, we cannot locate the referred $r_{1}$ and $r_{2}$ in Proposition 5 in the network when designing the credit scheme. Thus, the conditions for decentralizing SO flow pattern are theoretical but not practical. In fact, all the three papers considering transaction cost $[8,27,28]$ have consistently concluded that it is practically difficult to design a credit scheme decentralizing SO flow pattern when transaction cost is present.

\section{Solution Algorithm}

Under a given TCS, solving the credit price is a critical step to find the equilibrium flow pattern. Since the ME credit price is exactly the Lagrangian multiplier with respect to the credit conservation constraint, algorithms that can acquire solutions to both the primal and the dual problems are theoretically able to solve VI problem (12). In the literature, a gradient projection method was proposed by Han and Cheng [34] for solving equilibrium credit price and successfully applied in solving minimization model [35] and VI model [19]. In this section, a more efficient trial-and-error method is developed to solve the credit price in VI problem (12).

Essentially, a trial-and-error method is to find an equilibrium where the input equals the output when the analytical relationship between the two terms is uncertain. The trial-and-error method has been widely adopted in congestion pricing. Yang et al. [36] first developed a trialand-error implementation scheme of marginal-cost pricing on a general road network in the absence of demand functions. Then, Han and Yang [37] updated the scheme by reducing the trials needed for termination. Zhou et al. [38] proposed a trial-and-error congestion pricing scheme that addresses the capacity constraints as well. More recently, Guo et al. [39] proposed two trial-and-error operation schemes for the transport system when both the function of in-vehicle congestion costs and the distribution of perceived travel cost errors are unknown.

In the context of TCS, Wang and Yang [40] proposed a trial-and-error implementation of the tradable credit schemes for a single road. Later, the authors extended it to a general network case [41]. Different from the two existing works, which focus on solving equilibrium travel demand and optimal credit charge pattern, respectively, we propose a trial-and-error method for solving the equilibrium credit price in this paper.

In Yang et al.'s work [36], a trial-and-error method was proposed for the implementation of marginal-cost pricing in the absence of demand function. It inputs a target demand $d$ and outputs the observed demand $\bar{d}$ in each iteration. And it is proved that the input and the output can both converge to the system optimal (SO) solution $d^{\mathrm{SO}}$. In this example, the input as well as the output is the travel demand pattern, and the demand function relating the travel cost and travel demand is uncertain. In the system proposed in this paper, the uncertainty lies in the relationship between the link flow pattern and the resultant credit price. Thus, following the same trial-and-error fashion, we can iteratively input a credit price and solve an updated price as an output until the two matches with each other. In each iteration of the trial-and-error procedure, the method of successive averages (MSA) [42] is applied to solve the UE flow patterns, which is termed "inner iterations." Accordingly, the iterative updates on credit price is termed "outer iterations."

In the MSA, the move size $\alpha_{n}$ is not determined on the basis of some characteristics of the current solution. Instead, the sequence of move sizes $\alpha_{1}, \alpha_{2}, \ldots$ is determined priori. 
And the predetermined sequence of move sizes should satisfy the following two conditions:

$$
\begin{aligned}
0<\alpha_{n} \leq 1, \\
\sum_{n=1}^{\infty} \alpha_{n}=+\infty, \\
\sum_{n=1}^{\infty} \alpha_{n}^{2}<+\infty .
\end{aligned}
$$

Condition (45) represents the nonnegative move sizes. Conditions (46) and (47) guarantee that the algorithm will not stop prematurely and the variance of the variable will diminish as the iterations progress, respectively. A typical sequence of $\alpha_{n}$ is $\alpha_{n}=1 / n+1$. And to settle the nonmonotonic convergence using the relative change in link flow, the convergence measure on the flow in the last several iterations is applied, as shown in the following equation:

$$
\frac{\sqrt{\sum_{a \in A}\left(\bar{v}_{a}^{(n+1)}-\bar{v}_{a}^{(n)}\right)^{2}}}{\sum_{a \in A} \bar{v}_{a}^{(n)}} \leq \varepsilon_{0},
$$

where

$$
v_{a}^{(n)}=\frac{1}{m}\left(v_{a}^{(n)}+v_{a}^{(n-1)}+\cdots+v_{a}^{(n-m+1)}\right)=\frac{1}{m} \sum_{l=0}^{m-1} v_{a}^{(n-1)} .
$$

The details of the complete algorithm are given as follows:

Step 0. Determine the constant of tolerances $\varepsilon_{p}$ and $\varepsilon_{0}$ and the sequence of move sizes $\alpha_{n}=1 /(n+1)$. Let $\left[p_{a}(1)+p_{b}(1)\right]$ denote the initial search interval with $p_{a}(1)=0$, and $p_{b}(1)$ is a sufficiently large number ensuring that the ME credit price $p^{*}$ is within the interval. Set $p(1)=\left[p_{a}(1)+p_{b}(1)\right] / 2$.

Step 1. Run an MSA algorithm with $p(i)$.

(i) Initialization. Perform all-or-nothing assignments for each user class based on the set of initial travel disutility $c_{r, w}^{M^{(0)}}$ using equation (6) given $p=p(i)$, $v^{M}=0$, and $v=0$. This generates a set of link flow $\left(v^{M^{(1)}}, v^{(1)}\right)$. Set $n:=1$.

(ii) Update. $c_{r}^{m^{(n)}}=\sum_{a \in A} \beta^{m} t_{a}\left(v_{a}^{(n)}\right) \delta_{a, r}^{w}+p(i) e_{r}^{w}+T_{r}^{w}$ and get $c_{r, w}^{M^{(w)}}$.

(iii) Direction Finding. Perform all-or-nothing assignments for each user class based on the current set of travel disutility $c_{r, w}^{M^{(n)}}$ given $p=p(i)$, $v^{M}=v^{M^{(n)}}$, and $v=v^{(n)}$. This yields an auxiliary link flow pattern $\left(y^{M^{(n)}}, y^{(n)}\right)$.

(iv) Move. Find the new flow pattern $\left(v^{M^{(n+1)}}, v^{(n+1)}\right)$ by setting

$$
v^{M^{(n+1)}}=v^{M^{(n)}}+\alpha_{n}\left(y^{M^{(n)}}-v^{M^{(n)}}\right) .
$$

(v) Convergence Criterion. If the convergence criterion

$$
\sum_{m \in M} \frac{\sqrt{\sum_{a \in A}\left(\bar{v}_{a}^{m(n+1)}-\bar{v}_{a}^{m(n)}\right)}}{\sum_{a \in A} \bar{v}_{a}^{m(n)}} \leq \varepsilon_{0}
$$

is satisfied, stop the MSA algorithm and return $v^{M *}(i)=v^{M^{(n+1)}}, \quad v^{*}(i)=v^{(n+1)}$. Otherwise, set $n:=n+1$ and go to (ii).

Step 2. Check the scheme validity. If $\sum_{a \in A} \kappa_{a} v_{a}^{*}<K$, set $p$ $(i+1)=0$. Otherwise, calculate $\bar{p}(i)$ using equation (36). Let

$$
\begin{aligned}
& p_{a}(i+1)=\max \left\{p_{a}(i), \min (p(i), \bar{p}(i))\right\}, \\
& p_{b}(i+1)=\max \left\{p_{b}(i), \min (p(i), \bar{p}(i))\right\} .
\end{aligned}
$$

Then, set

$$
p(i+1)=\frac{1}{2}\left[p_{a}(i+1)+p_{b}(i+1)\right] .
$$

Step 3. If the convergence criterion

$$
|p(i+1)-p(i)| \leq \varepsilon_{p}
$$

is satisfied, stop the algorithm and return $p^{*}=p(i)$ and $p^{*}=p(i)$ and $\left(v^{M *}(i), v^{*}(i)\right)$. Otherwise, set $i:=i+1$ and go to Step 1 .

Next, we show that the algorithm is guaranteed to converge, and the convergence point exactly refers to the UE flow pattern $\left(v^{M *}, v^{*}\right)$ and ME credit price $p^{*}$.

First, we demonstrate some essential convergence properties of MSA as inner iteration.

Proposition 6. With a given credit price $p(i)$, the generated link flows by the iterative procedure have the following convergence properties:

$$
\begin{aligned}
\lim _{n \longrightarrow \infty} v^{M^{(n)}} & =v^{M *}(i), \\
\lim _{n \longrightarrow \infty} v^{(n)} & =v^{*}(i), \\
\lim _{n \longrightarrow \infty} \sum_{m \in M} \frac{\sqrt{\sum_{a \in A}\left(\bar{v}_{a}^{m(n+1)}-\bar{v}_{a}^{m(n)}\right)^{2}}}{\sum_{a \in A} \bar{v}_{a}^{m(n)}} & =0,
\end{aligned}
$$

where $\left(v^{M *}(i), v^{*}(i)\right)$ is the UE link flow pattern with the generalized travel cost function (6) in i-th iteration with $p(i)$.

Proof. It should be noted that in each inner iteration with MSA, the credit cost and transaction cost can be seen as constants in the generalized travel cost function (6), since the credit price and the trading volume are given before. Thus, the generalized travel cost $c_{r, w}^{m}$ is strictly convex in terms of link flows. With a descent vector $d^{(n)}=y^{M^{(n)}}-v^{M^{(n)}}$, the proposition of convergence regarding the method of successive averages [42] does hold for the link flow updating rule in equation (50). In other words, we have $\lim _{n \longrightarrow \infty} v^{(n)}=v^{*}(i)$ which naturally results in 
$\lim _{n \longrightarrow \infty} \sum_{m \in M}\left(\sqrt{\sum_{a \in A}\left(\bar{v}_{a}^{(n+1)}-\bar{v}_{a}^{m^{(n)}}\right)^{2}} / \sum_{a \in A} \bar{v}_{a}^{m^{(n)}}\right)=0$. This completes the proof.

According to Proposition 6, $\left(v^{M *}(i), v^{*}(i)\right)$ will be the solution flow pattern of VI problem (12) if $p(i)$ is the ME credit price. Then, we give the proof that the generated credit price by the outer iteration can converge to the ME unit credit price.

Proposition 7. The generated credit price by the iterative procedure can converge to the $M E$ credit price with $i \longrightarrow \infty$, i.e.,

$$
\lim _{i \longrightarrow \infty} p(i)=p^{*}
$$

Proof. We first show that for any $i$, it always holds that $p_{b}(i+1)-p_{a}(i+1) \leq\left(\left[p_{b}(i)-p_{a}(i)\right] / 2\right)$. If $p(i) \leq \bar{p}(i)$, then

$$
\begin{aligned}
& p_{b}(i+1)-p_{a}(i+1) \\
&= \min \left\{p_{b}(i), \max (p(i), \bar{p}(i))\right\} \\
&-\max \left\{p_{a}(i), \min (p(i), \bar{p}(i))\right\} \\
&= \min \left\{p_{b}(i), \bar{p}(i)\right\}-\max \left\{p_{a}(i), p(i)\right\} \\
& \leq p_{b}(i)-p(i)=\frac{1}{2}\left[p_{b}(i)-p_{a}(i)\right] . \\
& \text { If } p(i)>\bar{p}(i), \operatorname{then} \\
& p_{b}(i+1)-p_{a}(i+1) \\
&=\min \left\{p_{b}(i), \max (p(i), \bar{p}(i))\right\}-\max \left\{p_{a}(i), \min (p(i), \bar{p}(i))\right\} \\
&=\min \left\{p_{b}(i), p(i)\right\}-\max \left\{p_{a}(i), \bar{p}(i)\right\} \\
& \leq p(i)-p_{a}(i)=\frac{1}{2}\left[p_{b}(i)-p_{a}(i)\right] .
\end{aligned}
$$

Therefore, we have

$$
\begin{aligned}
p_{b}(i+1)-p_{a}(i+1) & \leq \frac{1}{2}\left[p_{b}(i)-p_{a}(i)\right] \\
& \leq \frac{1}{2^{i}}\left[p_{b}(1)-p_{a}(1)\right],
\end{aligned}
$$

which leads to

$$
\lim _{i \longrightarrow \infty}\left[p_{b}(i)-p_{a}(i)\right]=0 .
$$

Recall that $p(i)=\left(\left[p_{a}(i)+p_{b}(i)\right] / 2\right)$; we learn that $p(i)$ can always converge to a point, i.e.,

$$
\lim _{i \longrightarrow \infty} p_{a}(i)=\lim _{i \longrightarrow \infty} p_{b}(i)=\lim _{i \longrightarrow \infty} p(i)=p_{0} .
$$

When convergence is achieved, the convergence point $p(i)=p_{0}$ and corresponding $\left(v^{M *}(i), v^{*}(i)\right)$ exactly satisfy $\mathrm{ME}$ conditions and UE conditions. This implies the system is at equilibrium. Due to the uniqueness of credit price at equilibrium, it can be referred that the convergence point $p_{0}$ must be the ME credit price $p^{*}$, i.e., $\lim _{i \rightarrow \infty} p(i)=p^{*}$. This completes the proof.

\section{Numerical Examples and Findings}

5.1. The Computational Efficiency of the Solution Algorithm. In this subsection, we adopt two networks shown in Figure 1 to demonstrate the convergence properties of the proposed algorithm. The Sioux Falls network is a small network consisting of 24 nodes, 76 links, and 528 O-D pairs. The Anaheim network is a midsize network consisting of 416 nodes, 914 links, and 1406 O-D pairs. The relevant network data were taken from the transportation network datasets maintained by Stabler et al. [43].

To compare the bisection-based trial-and-error method with traditional one, we solve model (12) for the two networks by the gradient projection method (the algorithm is given in Appendix A) and the proposed method, respectively. Both algorithms terminate based on the difference of credit prices in successive iterations.

We assume two user classes with $\beta^{1}=1$ and $\beta^{2}=2$, and they are uniformly distributed to all the O-D pairs with the proportion of $60 \%$ and $40 \%$, respectively. The link travel times are calculated from the Bureau of Public Road (BPR) function as below:

$$
t_{a}=t_{a}^{0}\left(1+0.15\left(\frac{v_{a}}{C_{a}}\right)^{4}\right), \quad \forall a \in A
$$

where $t_{a}^{0}, a \in A$ is the link free flow travel time and $C_{a}, a \in A$ is the link capacity. Further, we assume that each traveler gets 5 credits initially and set the credit charge on each link as the difference between the marginal social cost and the marginal private cost at SO status, i.e.,

$$
\kappa_{a}=\left.v_{a} \frac{\mathrm{d} t_{a}\left(v_{a}\right)}{\mathrm{d} v_{a}}\right|_{v_{a}=\tilde{v}_{a}}, \quad \forall a \in A,
$$

and set $\eta=1, \rho=0.1$.

Figure 2 shows the convergence results of the two methods. From the figure, it is clear that the number of iterations needed for termination of the proposed algorithm is considerably less than that of the gradient projection method. From Table 1, we can also see that the bisection-based trialand-error method can save a half of time for Sioux Falls network and about $87.5 \%$ for Anaheim network, indicating that the proposed method far outperforms the traditional one.

The computational efficiency of the proposed method may be explained by higher rate of convergence of the bisection-based method as well as the use of equation (36) in the algorithm. In other words, the calculation of the credit price in each iteration fully explores the information acquired from the flow pattern. In contrast, the projection-based method does not explore any information from equation (36), and instead, it updates based on the total charged credits, which is much more general.

5.2. The Significance of considering Heterogenous VOT. Then, a toy network is adopted in the subsequent analysis to present more detailed findings (according to equation (6), the trading volume can only be acquired when the path flow pattern is obtainable; hence, a large-scale network cannot be 


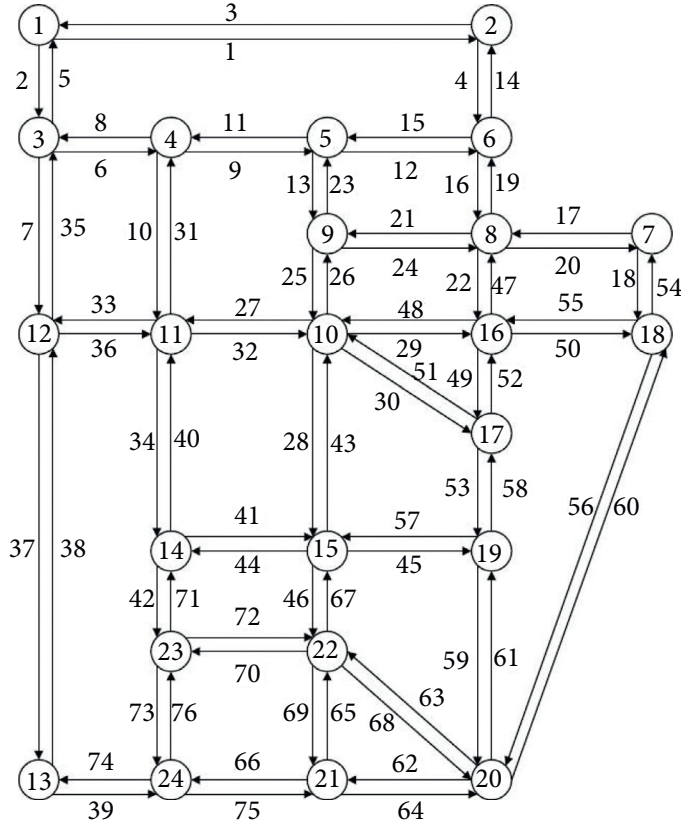

(a)

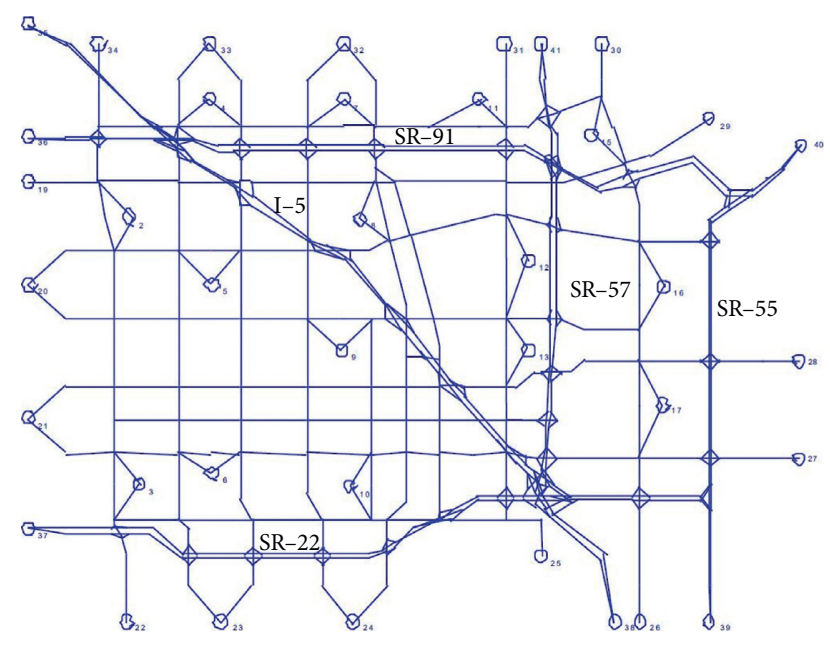

(b)

Figure 1: The two test networks. (a) The Sioux Falls network. (b) The Anaheim network.

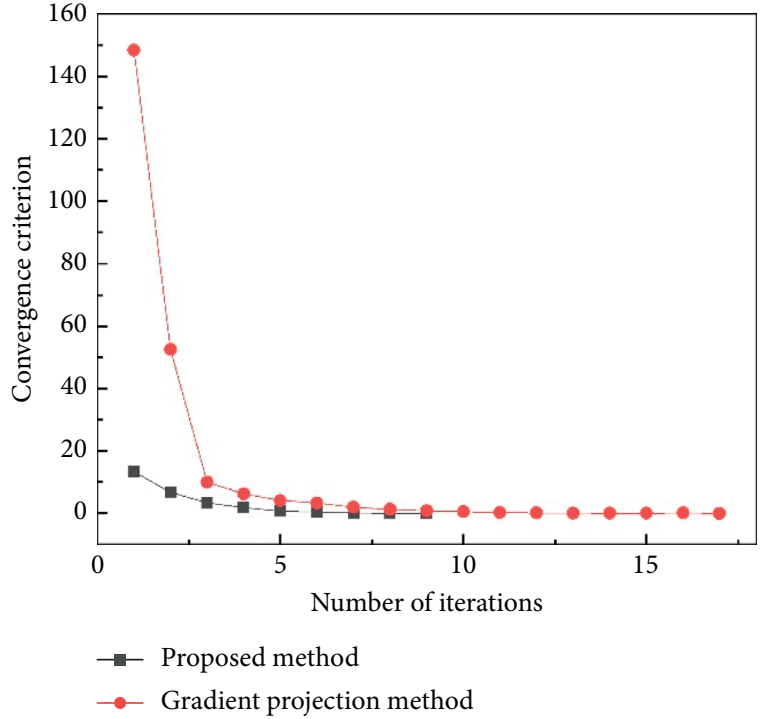

(a)

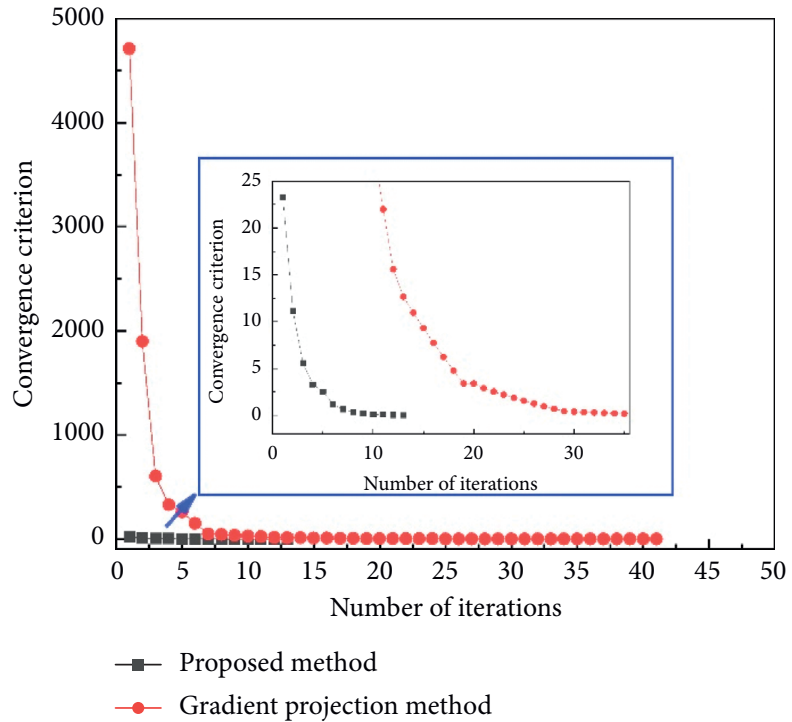

(b)

Figure 2: Convergence plots on the two example networks. (a) Results on Sioux Falls network. (b) Results on Anaheim network.

TABLE 1: CPU times for the two methods in solving the VI model on example networks.

\begin{tabular}{lcc}
\hline Solution method & Example network & Anaheim (s) \\
\hline Gradient projection & 167.3 & 5015.3 \\
Bisection-based trial-and-error & 82.6 & 626.8 \\
\hline
\end{tabular}




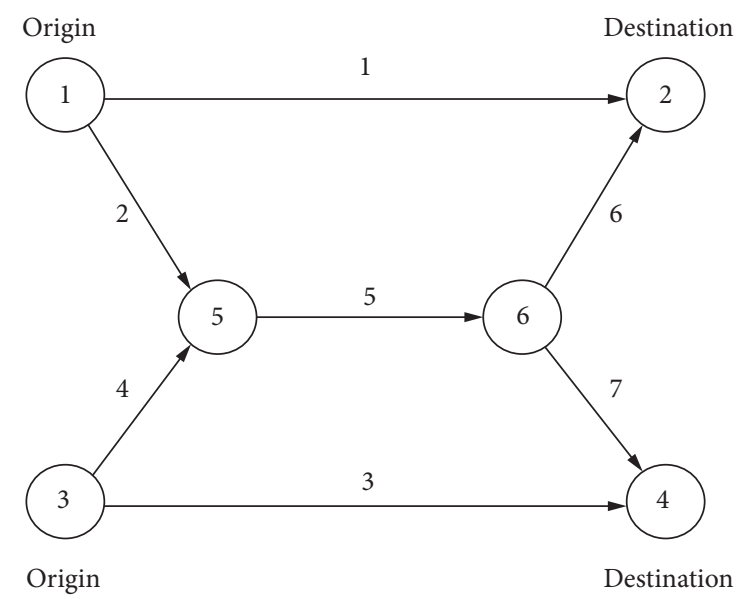

Figure 3: The toy network.

applied to investigate the variation in the trading volume; besides, it is also much easier to capture the travel costs of different user classes; therefore, a small network is adopted in the subsequent analysis).

The example network, as shown in Figure 3, consists of six nodes, seven links, two O-D pairs: O-D pair 1 (node $1 \longrightarrow$ node 2 ) and O-D pair 2 (node $3 \longrightarrow$ node 4 ), and four paths: path 1 (link 1), path 2 (link 2-5-6), path 3 (link 4-5-7), and path 4 (link 3). The information of the network and credit scheme is given in Table 2 .

We assume that there are three user classes with $\beta^{1}=1$, $\beta^{2}=2$, and $\beta^{3}=3$. The travel demands for the three user classes are 30, 20, and 10 units for O-D pair 1 and 30, 10, and 10 units for O-D pair 2, respectively. We set the user-anonymous credit scheme consistent with that in [28], where the total amount of distributed credits is 660 , and each traveler on the network is initially distributed 6 credits.

Given the information above, we first examine the significance of considering heterogenous VOT. With $\eta=1$, we solve the equilibrium credit price and system travel time for three cases with homogenous VOT as 1, 1.64 (it is the weighted average of the proposed VOT profile over travel demands, which can be calculated as $(1 \times 60+2 \times 30+3 \times 20)(60+50)=1.64)$, and 3 , respectively, and compare them with the heterogenous case. The results are shown in Figure 4.

From Figure 4(a), it is clear that excluding user heterogeneity leads to an underestimation of the system travel time, and the impact of transaction cost is considerably less significant in the homogenous cases. Furthermore, it can be seen from Figure 4(b) that the equilibrium credit price increases with the marginal transaction cost in homogenous cases, while the trend in the heterogenous case is the opposite. The diversity between the homogenous and heterogenous cases can be explained by the varying weights on travel time for different user classes, and such differences verify the significance of considering user heterogeneity in the equilibrium problem.
TABLE 2: Input data of the small network.

\begin{tabular}{lccc}
\hline Link & Free flow travel time & Capacity & Credit charge \\
\hline 1 & 10 & 35 & 9 \\
2 & 3 & 30 & 2 \\
3 & 12 & 35 & 8 \\
4 & 4 & 35 & 1 \\
5 & 5 & 35 & 1 \\
6 & 3 & 35 & 2 \\
7 & 4 & 25 & 1 \\
\hline
\end{tabular}

5.3. The Impact of Transaction Cost. Next, we move on to explore the impacts of transaction costs using the same toy network.

5.3.1. The Impact of Transaction Cost on Credit Market. The impact on the credit market can be sufficiently reflected by the total trading volume and the resultant credit price. We solve the proposed model for the three kinds of markets discussed in Section 2.1 (i.e., a trading-encouraging market for $\eta<1$, a trading-controlling for $\eta>1$, and a moderate market for $\eta=1$ ). The results are shown in Figure 5 .

From the figure, it can be observed that the increase in $\rho$ brings a drop in trading volume in all cases. However, the decrease in the trading-encouraging market is very limited, which is exactly due to the monotonically decreasing marginal transaction cost with respect to the trading volume. In other words, the inhibition of transaction cost on credit trading is largely compensated by the "discounts" over quantity in the market.

In terms of the credit price, we can see that there is no remarkable change in the credit price in the tradingencouraging market as well. For the other two markets, it is noted that the changing patterns are roughly consistent, where the credit price decreases at first and then turns to an increasing trend halfway at some values of $\rho$. This is inconsistent with the previous work indicating that the increase in transaction cost could suppress trading volumes and thus reduce credit price considering homogenous travelers on the network [27]. To figure out the factors attributing to the counterintuitive increase in credit price, we further investigate the trading volumes of different user classes, as shown in Figure 6.

Interestingly, we find that the value of $\rho$ after which the credit price swerves to an increasing trend is exactly the turning point where the credit demand for user class 3 exceeds that for user class 2 (see the curves $\eta=1,2$ in Figures 5(b) and 6). This implies that when the credit demand for users with the highest VOT becomes dominant, the credit price can increase with the transaction cost even though credit trading has been suppressed.

5.3.2. The Impact of Transaction Cost on Individual Travel Costs. To investigate the impact of transaction cost on individual travel cost, we solve the user-equilibrium travel cost 


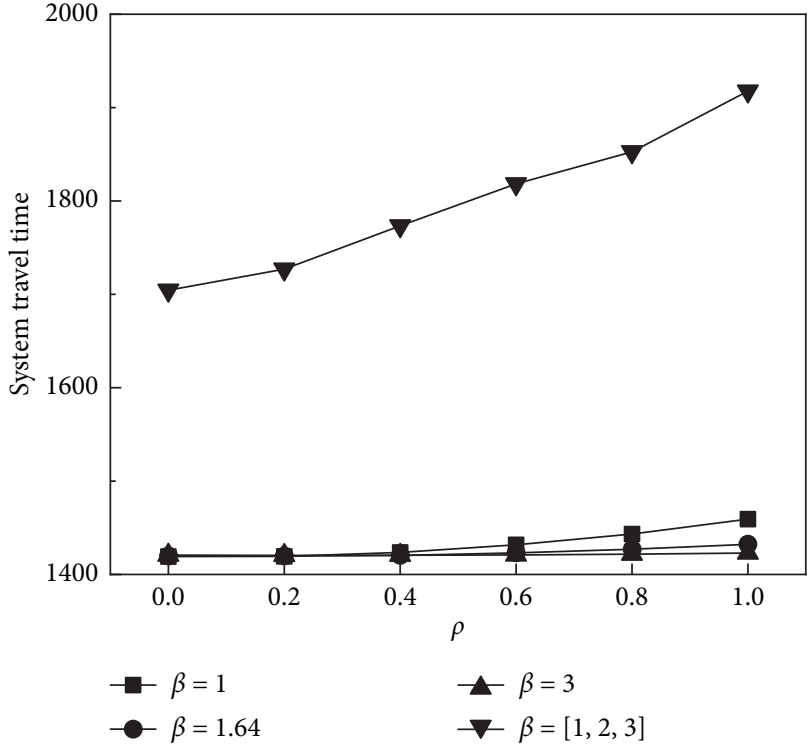

(a)

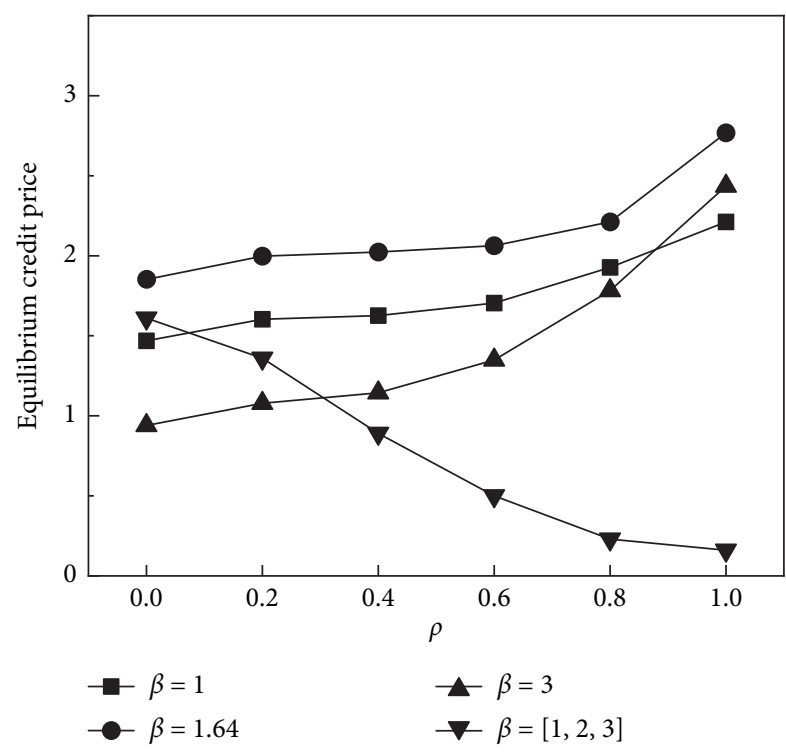

(b)

Figure 4: Variations in trading volume and credit price with different types of transaction costs. (a) Trading volume. (b) Credit price.

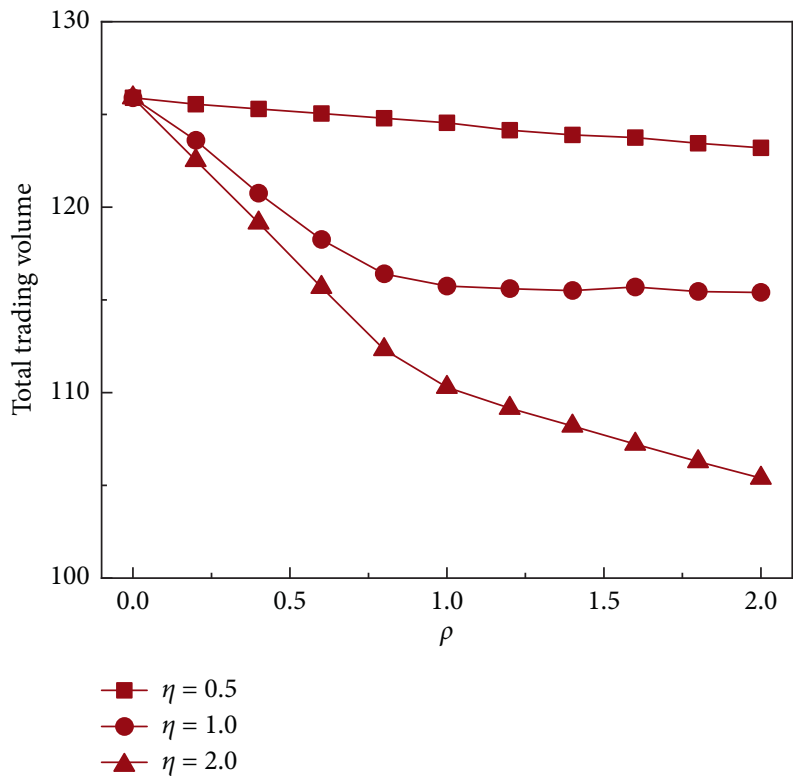

(a)

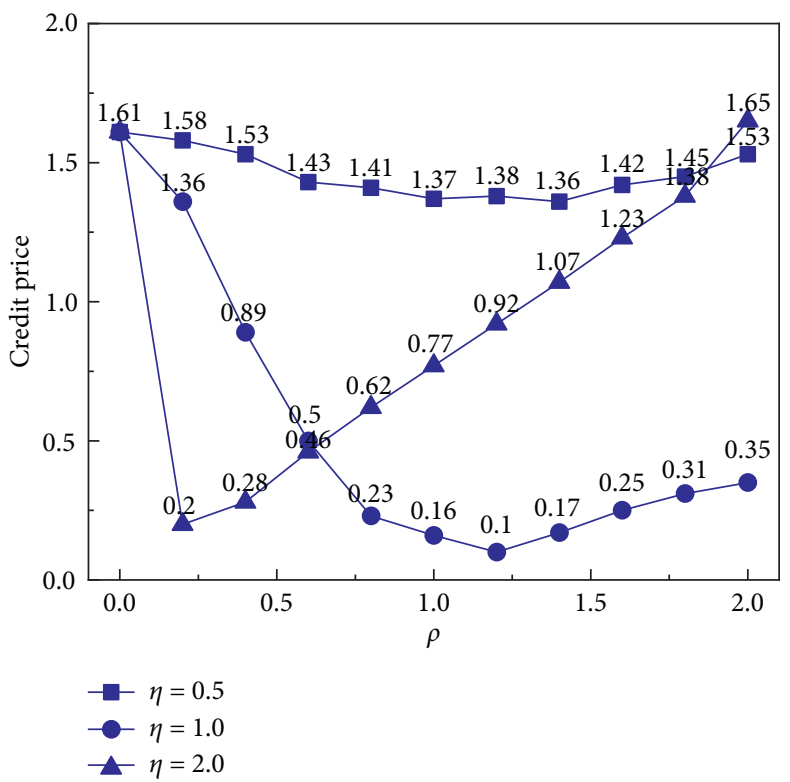

(b)

FIGURE 5: Variations in trading volume and credit price with different types of transaction costs. (a) Trading volume. (b) Credit price.

excluding the TCS as a benchmark and compare it with the cases under the scheme.

Figure 7 shows the variations in better-off degree of individual monetary travel cost in different kinds of markets. The main findings are listed as below.

First, we find that user classes 2 and 3 can always benefit from the TCS when $\eta=0.5$, while user class 1 can gain benefits only when $\rho \leq 0.4$. In other words, a Pareto-improving can be achieved given a sufficiently small $\rho$ in the trading- encouraging market. By comparison, in the trading-controlling market, the travel utility for user class 1 can be deteriorated even when $\rho$ is less than 0.1 , meaning that it is practically difficult to achieve a Pareto-improving in such case.

On the other hand, it is interesting to note that the users with the highest VOT enjoy the most benefits brought by the TCS, and these users can always experience a reduction in travel cost in each of the three markets. Moreover, taking a 


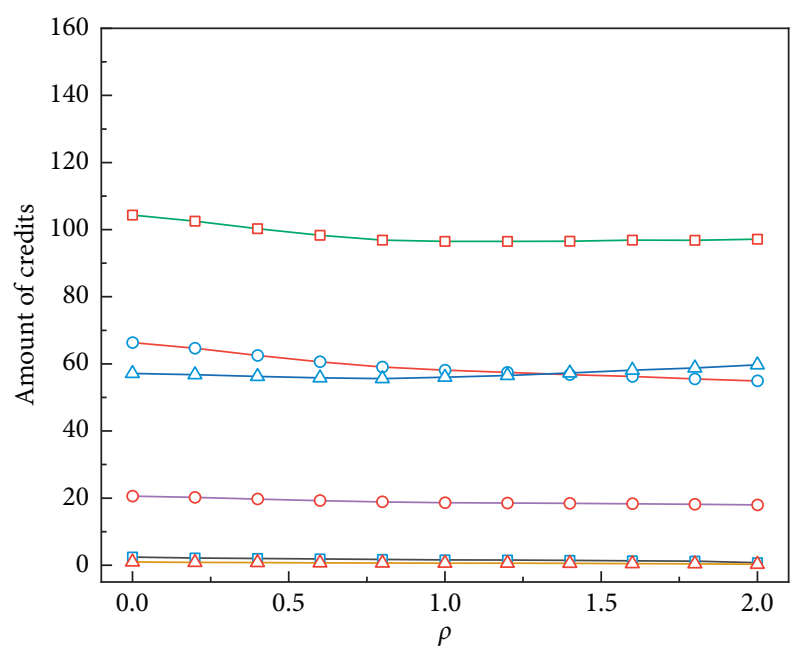

$\neg-$ Credit demand for user class 1

$-\circ$ Credit demand for user class 2

$\triangle$ Credit demand for user class 3

$-\square-$ Credit supply for user class 1

- - Credit supply for user class 2

$\triangle$ Credit supply for user class 3

(a)

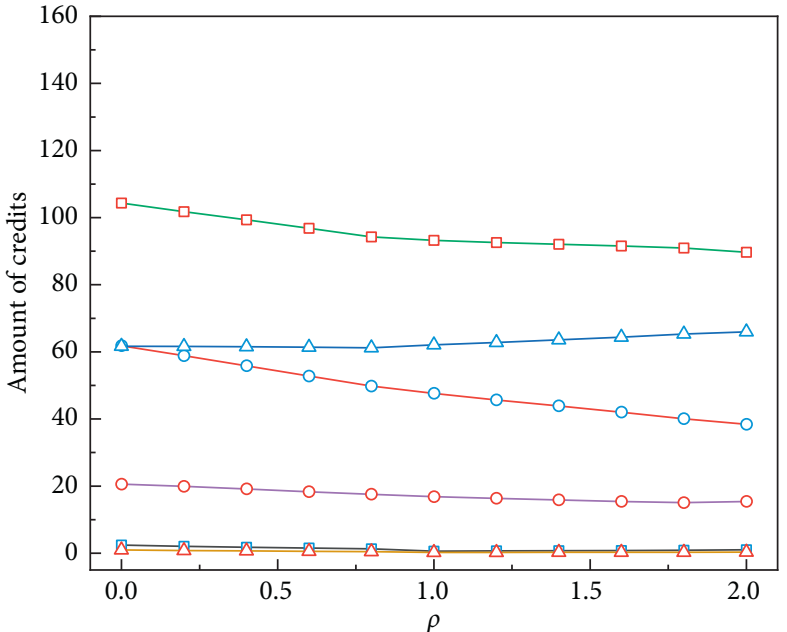

$-\square$ Credit demand for user class 1

$-\infty$ Credit demand for user class 2

$\triangle$ Credit demand for user class 3

$-\square-$ Credit supply for user class 1

- - Credit supply for user class 2

$\triangle$ Credit supply for user class 3

(b)

FIgURE 6: Trading volumes of different user classes. (a) $\eta=1$. (b) $\eta=2$.

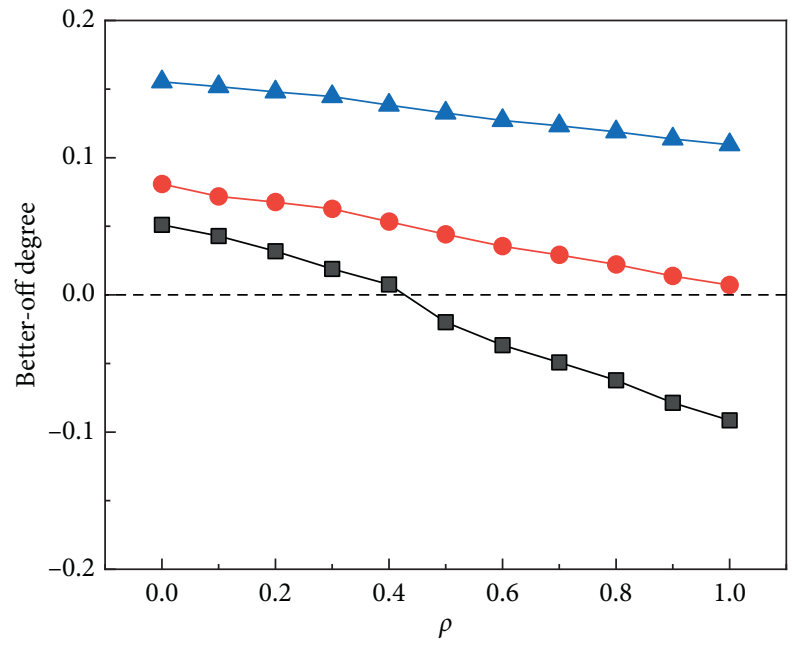

User class 1

User class 2

- User class 3

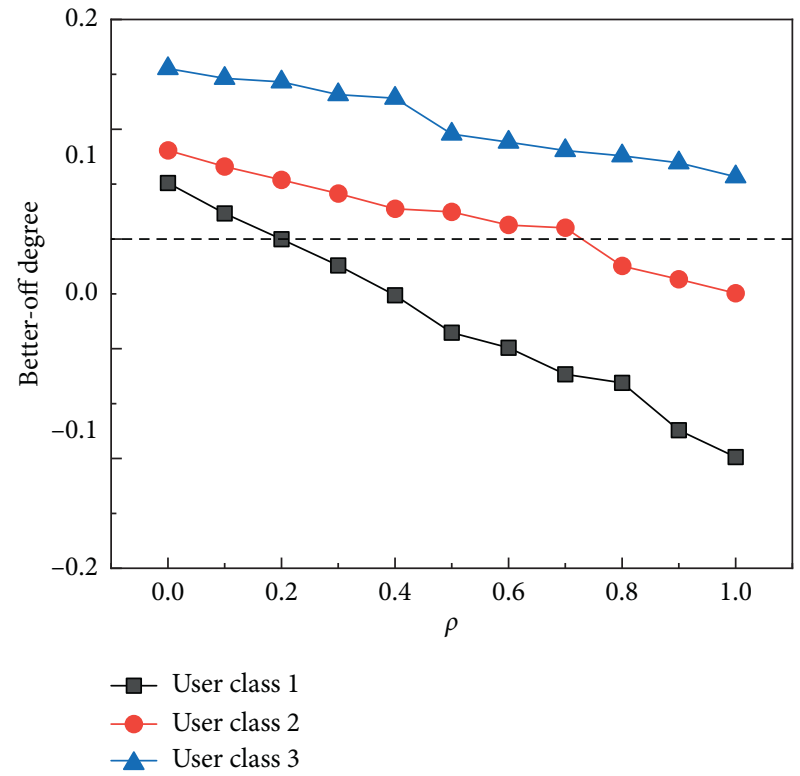

(b)

Figure 7: Continued. 


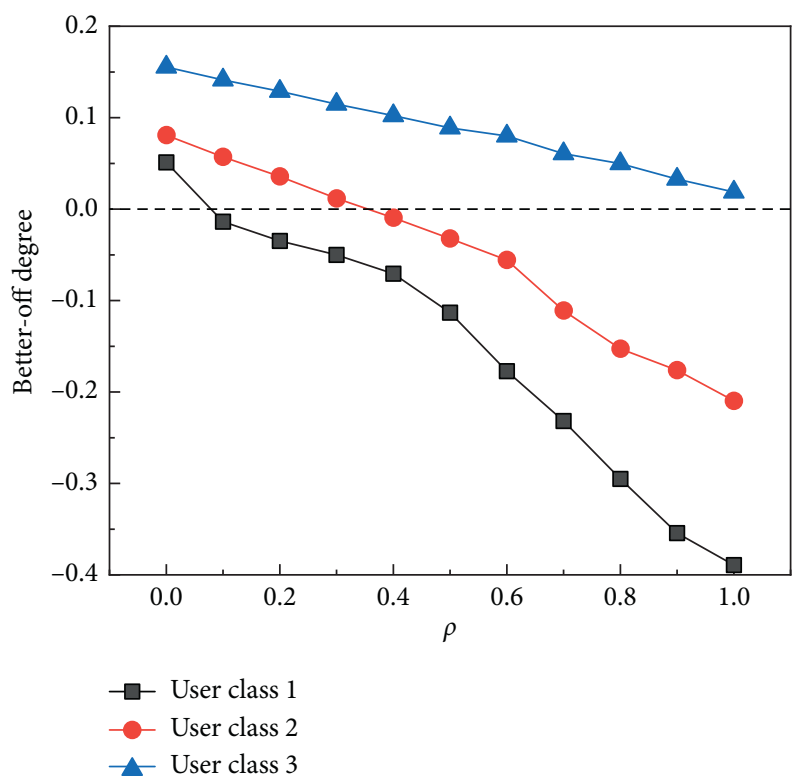

(c)

FiguRE 7: Variations in better-off degree with different types of transaction costs. (a) Better-off degree with $\eta=0.5$. (b) Better-off degree with $\eta=1.0$. (c) Better-off degree with $\eta=2.0$.

close look at the slopes of the three curves, we find that the marginal contribution of increasing $\rho$ to the reduction in better-off degree declines with VOT, meaning that users with the lowest VOT suffer most from a unit change in transaction cost.

To sum up, the trading volume and equilibrium credit price are relatively insensitive to parameter $\rho$ in the tradingencouraging market, while there are remarkable changes in the other two markets. Besides, users with the higher VOT are more likely to experience a reduction in travel cost with the implementation of TCS even though transaction cost is considered. Meanwhile, those with the lowest VOT suffer the most from the increase in transaction cost in all the three markets, and it is extremely difficult to achieve a Paretoimproving in the trading-controlling market.

\section{Conclusions and Extensions}

In this paper, the impacts of transaction cost are investigated under a tradable credit scheme considering user heterogeneity. We consider a multiclass tradable credit scheme characterized by user-anonymous O-D-specific credit distribution and link-specific credit charge and incorporate transaction cost which is associated with trading volume and independent of credit price. Three different kinds of credit-trading markets, which are defined based on the monotonicity of the marginal transaction cost, are considered. We formulate the UE and ME problems as a VI problem with existence of solutions guaranteed. A novel bisection-based trial-and-error method is proposed to solve the problem. Based on the results from the example networks, we first demonstrate the convergence efficiency of the proposed algorithm and the significance of considering user heterogeneity in the equilibrium problem. Then, we discuss the impacts of transaction cost on the functioning of credit market as well as the individual travel cost. The main findings are given as follows:

(i) The trading volume and equilibrium credit price are relatively insensitive to the change of transaction cost in the trading-encouraging market.

(ii) In the moderate and the trading-controlling market, trading volume is observed to be suppressed significantly as the transaction cost increases. Moreover, when the credit demand for users with the highest VOT becomes dominant, the credit price can increase with the transaction cost even though credit trading has been suppressed.

(iii) Users with the higher VOT are more likely to experience a reduction in travel cost with the implementation of TCS, even though transaction cost is considered. Meanwhile, those with the lowest VOT always suffer the most from the increase in transaction cost in all the three markets.

(iv) A Pareto-improving can be achieved given a sufficiently small marginal transaction cost in the trading-encouraging market. However, it is practically difficult to achieve a Pareto-improving in the trading-controlling market.

Unfortunately, although we establish the conditions for SO credit scheme design in presence of transaction cost, it is practically difficult in reality. Future research 
studies can be carried out in designing tradable credit scheme for other objectives, such as minimizing the efficiency loss caused by the transaction cost. And the discrete set of VOT can be generalized to a continuous distribution, which can relax the assumption in Proposition 3 as well as the conditions for uniqueness of credit price [2]. Besides, the assumption of fixed demand can be relaxed to the elastic demand case. In such a situation, the impacts of transaction cost on travel demand are considered and a more realistic equilibrium flow pattern is expected to be acquired.

\section{Appendix}

\section{A. The Algorithm of the Gradient Projection Method}

The gradient projection method for solving the equilibrium credit price is listed as below.

Step 0. Determine the constant of tolerance $\varepsilon_{p}$ and the sequence of move sizes $\alpha n$ given by equations (46)-(49). Randomly initialize the credit price $p(i)>0$. Set $i:=1$.

Step 1. Run an MSA algorithm with $p(i)$ and obtain the equilibrium flow pattern $v^{M *}(i)$ and $v^{*}(i)$ (the procedure is consistent with that in Section 4).

Step 2. Check the scheme validity. If $\sum_{a \in A} \kappa_{a} v_{a}^{*}(i)<K$, set $p(i+1)=0$. Otherwise, calculate $p(i+1)$ by $p(i+1)=\max \left(0, p(i)+(1 / n)\left(\sum_{a \in A} \kappa_{a} v_{a}^{*}(i)-K\right)\right)$.

Step 3. If the convergence criterion

$$
|p(i+1)-p(i)| \leq \varepsilon_{p}
$$

is satisfied, stop the algorithm and return $p^{*}=p(i)$ and $\left(v^{M *}, v^{*}\right)=\left(v^{M *}(i), v^{*}(i)\right)$. Otherwise, set $i:=i+1$ and go to Step 1 .

\section{Data Availability}

Data about the Sioux Falls and the Anaheim networks were obtained from the GitHub repository "Transportation Networks for Research" (https://github.com/bstabler/ TransportationNetworks).

\section{Conflicts of Interest}

The authors declare that there are no conflicts of interest regarding the publication of this paper.

\section{Acknowledgments}

This research was supported by the Postgraduate Research \& Practice Innovation Program of Jiangsu Province (No. SJCX20_0016) and the Fundamental Research Funds for the Central Universities.

\section{References}

[1] H. Yang and X. Wang, "Managing network mobility with tradable credits," Transportation Research Part B: Methodological, vol. 45, no. 3, pp. 580-594, 2011.

[2] X. Wang, H. Yang, D. Zhu, and C. Li, "Tradable travel credits for congestion management with heterogeneous users," Transportation Research Part E: Logistics and Transportation Review, vol. 48, no. 2, pp. 426-437, 2012.

[3] D.-L. Zhu, H. Yang, C.-M. Li, and X.-L. Wang, "Properties of the multiclass traffic network equilibria under a tradable credit scheme," Transportation Science, vol. 49, no. 3, pp. 519-534, 2015.

[4] L.-J. Tian, H. Yang, and H.-J. Huang, "Tradable credit schemes for managing bottleneck congestion and modal split with heterogeneous users," Transportation Research Part E: Logistics and Transportation Review, vol. 54, pp. 1-13, 2013.

[5] J.-P. Wang, T.-L. Liu, and H.-J. Huang, "Tradable OD-based travel permits for bi-modal traffic management with heterogeneous users," Transportation Research Part E: Logistics and Transportation Review, vol. 118, pp. 589-605, 2018.

[6] M. Miralinaghi and S. Peeta, "A multi-period tradable credit scheme incorporating interest rate and traveler value-of-time heterogeneity to manage traffic system emissions," Frontiers in Built Environment, vol. 4, 2018.

[7] D. Wu, Y. Yin, S. Lawphongpanich, and H. Yang, "Design of more equitable congestion pricing and tradable credit schemes for multimodal transportation networks," Transportation Research Part B: Methodological, vol. 46, no. 9, pp. 1273-1287, 2012.

[8] F. He, Y. Yin, N. Shirmohammadi, and Y. Nie, "Tradable credit schemes on networks with mixed equilibrium behaviors," Transportation Research Part B: Methodological, vol. 57, pp. 47-65, 2013.

[9] M. Miralinaghi, S. Peeta, X. He, and S. V. Ukkusuri, "Managing morning commute congestion with a tradable credit scheme under commuter heterogeneity and market loss aversion behavior," Transportmetrica B: Transport Dynamics, vol. 7, no. 1, pp. 1780-1808, 2019.

[10] N. Shirmohammadi, M. Zangui, Y. Yin, and Y. Nie, "Analysis and design of tradable credit schemes under uncertainty," Transportation Research Record: Journal of the Transportation Research Board, vol. 2333, no. 1, pp. 27-36, 2013.

[11] N. Shirmohammadi and Y. Yin, "Tradable credit scheme to control bottleneck queue length," Transportation Research Record: Journal of the Transportation Research Board, vol. 2561, no. 1, pp. 53-63, 2016.

[12] G. Wang, Z. Gao, M. Xu, and H. Sun, "Models and a relaxation algorithm for continuous network design problem with a tradable credit scheme and equity constraints," Computers \& Operations Research, vol. 41, pp. 252-261, 2014.

[13] M. Miralinaghi and S. Peeta, "Multi-period equilibrium modeling planning framework for tradable credit schemes," Transportation Research Part E: Logistics and Transportation Review, vol. 93, pp. 177-198, 2016.

[14] M. Miralinaghi and S. Peeta, "Promoting zero-emissions vehicles using robust multi-period tradable credit scheme," Transportation Research Part D: Transport and Environment, vol. 75, pp. 265-285, 2019.

[15] H. Wang and X. Zhang, "Joint implementation of tradable credit and road pricing in public-private partnership networks considering mixed equilibrium behaviors," Transportation Research Part E: Logistics and Transportation Review, vol. 94, pp. 158-170, 2016. 
[16] Y. Bao, Z. Gao, H. Yang, M. Xu, and G. Wang, "Private financing and mobility management of road network with tradable credits," Transportation Research Part A: Policy and Practice, vol. 97, pp. 158-176, 2017.

[17] G. Wang, M. Xu, S. Grant-Muller, and Z. Gao, "Combination of tradable credit scheme and link capacity improvement to balance economic growth and environmental management in sustainable-oriented transport development: a bi-objective bi-level programming approach," Transportation Research Part A: Policy and Practice, vol. 137, p. 459, 2020.

[18] G. Wang, Z. Gao, and M. Xu, "Integrating link-based discrete credit charging scheme into discrete network design problem," European Journal of Operational Research, vol. 272, no. 1, pp. 176-187, 2019.

[19] F. Zhang, J. Lu, and X. Hu, "Traffic equilibrium for mixed traffic flows of human-driven vehicles and connected and autonomous vehicles in transportation networks under tradable credit scheme," Journal of Advanced Transportation, vol. 2020, Article ID 8498561, 18 pages, 2020.

[20] S. E. Seilabi, M. T. Tabesh, A. Davatgari, M. Miralinaghi, and S. Labi, "Promoting autonomous vehicles using travel demand and lane management strategies," Frontiers in Built Environment, vol. 6, p. 6, 2020.

[21] N. Dogterom, Y. Bao, M. Xu, and D. Ettema, "Acceptability of a tradable driving credit scheme in the Netherlands and Beijing," Case Studies on Transport Policy, vol. 6, no. 4, pp. 499-509, 2018.

[22] M. Xu and S. Grant-Muller, "Trip mode and travel pattern impacts of a tradable credits scheme: a case study of Beijing," Transport Policy, vol. 47, pp. 72-83, 2016.

[23] N. Dogterom, D. Ettema, and M. Dijst, "Behavioural effects of a tradable driving credit scheme: results of an online stated adaptation experiment in the Netherlands," Transportation Research Part A: Policy and Practice, vol. 107, pp. 52-64, 2018.

[24] Y. Tian, Y.-C. Chiu, and J. Sun, "Understanding behavioral effects of tradable mobility credit scheme: an experimental economics approach," Transport Policy, vol. 81, pp. 1-11, 2019.

[25] S. Grant-Muller and M. Xu, "The role of tradable credit schemes in road traffic congestion management," Transport Reviews, vol. 34, no. 2, pp. 128-149, 2014.

[26] N. Dogterom, D. Ettema, and M. Dijst, "Tradable credits for managing car travel: a review of empirical research and relevant behavioural approaches," Transport Reviews, vol. 37, no. 3, pp. 322-343, 2017.

[27] Y. Nie, "Transaction costs and tradable mobility credits," Transportation Research Part B: Methodological, vol. 46, no. 1, pp. 189-203, 2012.

[28] Y. Bao, Z. Gao, M. Xu, and H. Yang, "Tradable credit scheme for mobility management considering travelers' loss aversion," Transportation Research Part E: Logistics and Transportation Review, vol. 68, pp. 138-154, 2014.

[29] F. Zhang, J. Lu, and X. Hu, "Tradable credit scheme design with transaction cost and equity constraint," Transportation Research Part E: Logistics and Transportation Review, vol. 145, Article ID 10213, 2021.

[30] T. N. Cason and L. Gangadharan, "Transactions costs in tradable permit markets: an experimental study of pollution market designs," Journal of Regulatory Economics, vol. 23, no. 2, pp. 145-165, 2003.

[31] S. Kerr and D. C. Maré, "Transaction costs and tradable permit markets: the United States lead phasedown," in Proceedings of the 2011 Working Paper, Motu Economic and Public Policy Research, Wellington, New Zealand, 2011.
[32] F. Facchinei and J. S. Pang, Finite-Dimensional Variational Inequalities and Complementarity Problems, Springer-Verlag, Berlin, Germany, 2003.

[33] M. J. Smith, "The existence, uniqueness and stability of traffic equilibria," Transportation Research Part B: Methodological, vol. 13, no. 4, pp. 295-304, 1979.

[34] F. Han and L. Cheng, "Stochastic user equilibrium model and its algorithm considering tradable credit scheme," Journal of Southeast University, vol. 46, pp. 215-220, 2016.

[35] F. Han and L. Cheng, "Stochastic user equilibrium model with a tradable credit scheme and application in maximizing network reserve capacity," Engineering Optimization, vol. 49, no. 4, pp. 549-564, 2017.

[36] H. Yang, Q. Meng, and D.-H. Lee, "Trial-and-error implementation of marginal-cost pricing on networks in the absence of demand functions," Transportation Research Part B: Methodological, vol. 38, no. 6, pp. 477-493, 2004.

[37] D. Han and H. Yang, "Congestion pricing in the absence of demand functions," Transportation Research Part E: Logistics and Transportation Review, vol. 45, no. 1, pp. 159-171, 2009.

[38] B. Zhou, M. Bliemer, H. Yang, and J. He, "A trial-and-error congestion pricing scheme for networks with elastic demand and link capacity constraints," Transportation Research Part B: Methodological, vol. 72, pp. 77-92, 2015.

[39] R.-Y. Guo, W. Y. Szeto, and J. Long, "Trial-and-error operation schemes for bimodal transport systems," Transportation Research Part B: Methodological, vol. 131, pp. 106-123, 2020.

[40] X. Wang and H. Yang, "Bisection-based trial-and-error implementation of marginal cost pricing and tradable credit scheme," Transportation Research Part B: Methodological, vol. 46, no. 9, pp. 1085-1096, 2012.

[41] X. Wang, H. Yang, D. Han, and W. Liu, "Trial and error method for optimal tradable credit schemes: the network case," Journal of Advanced Transportation, vol. 48, no. 6, pp. 685-700, 2014.

[42] W. B. Powell and Y. Sheffi, "The convergence of equilibrium algorithms with predetermined step sizes," Transportation Science, vol. 16, no. 1, pp. 45-55, 1982.

[43] B. Stabler, H. Bar-Gera, and E. Sall, "Transportation networks for research,” 2018, https:/github.com/bstabler/ transportationnetworks. 